\title{
State identification of underdetermined grids
}

\author{
Martin Wolter \\ Leibniz Universität Hannover
}

Germany

\section{Introduction}

Methods of grid state identification are mathematical algorithms to obtain information on state variables of electric power system grids. These procedures are based fundamentally on the acquisition of measured, forecasted or estimated values, which are universally referred to as influencing variables, or, more simply, as measurands. In combination with knowledge of grid topology and electrical equipment parameters, identification methods aim at the best possible determination of the grid state.

Depending on the ratio of the number of available independent measurands and the quantity of state variables, the equation system to be solved is either overdetermined, determined or underdetermined. Thus, different procedures of grid state identification are applied.

Independent of the identification approach the basic proceeding of grid state determination remains the same. Starting at an initial guess the grid state identificator returns a nodal voltage vector which is used to calculate secondary grid values like nodal currents, nodal powers or terminal currents and powers. Calculation results are compared to the available information on influencing variables. The grid state identificator now iteratively adjusts the voltages to minimize the difference between measured and calculated values.

If the input vector is redundant and contains more (real-valued) measurands than twice the number of nodes, the equation system is overdetermined. This sort of problem is characteristic of high voltage and ultra high voltage transmission grids. Recording of more measurands than needed for uniquely solving the equation system is used to protect grid state identification against measurement uncertainties and outages of measurement facilities.

Typically, the number of measurands is more than twice as high as the amount which would have been necessary to uniquely calculate a grid state. To solve an overdetermined equation system state estimation algorithms based on Gaussian Least-Squares-Optimization or artificial neural networks are applied which find a solution vector of nodal voltages that minimizes the mean square error.

If the number of independent (real-valued) measurands is exactly twice the number of nodes, the equation system is uniquely solvable. In case of measuring only voltages and currents, a direct solution without any iteration is possible. If powers are used as influencing variables, an iterative solution approach is applied using a Newton-Raphson or fixed-point 
iteration method. The special case of using solely nodal powers as influencing variables is called power flow calculation.

If the input vector contains less real-valued measurands than twice the number of nodes, the equation system is underdetermined. This sort of problem is characteristic of medium and low voltage distribution grids due to in the past exact knowledge of the grid state was unnecessary. So, installation of measurement facilities was not enforced to save costs. Nowadays, changed basic conditions necessitate adequate knowledge of equipment utilization. Unfortunately, underdetermined equation systems are not uniquely solvable but offer an infinite number of solutions instead. Thus, estimation approaches are needed to either limit the solution space or to estimate the most probable grid state.

\section{Motivation}

Considering particularly distribution grids, in a liberalized market the behavior and utilization of the electric power system is strongly influenced by the interests of market participants, namely on the one hand producers and consumers of energy and on the other hand the grid operators. The interests of all parties are mostly driven by economical considerations.

It is obvious that the goals of different market participants are divergent. As a consequence, energy management systems (EMS) are developed to economically combine all objectives. They are supposed to find strategies for grid operation and utilization which are convenient for all involved parties.

Decentralized energy management systems offer concepts to local utilities to face future challenges under changing conditions of the energy sector. Therefore, the energy management covers all the processes prospectively involved in the future of distributed energy supply to have the continuing ability to safely operate distribution grids and to securely, effectively and economically supply customers. This includes methods to optimize power acquisition as well as strategies to maintain and improve power quality.

On the one hand the above mentioned aims are achieved by influencing load and generation i.e. by optimizing assignment of feeders and adaptable loads taking into account technical and economical constraints.

On the other hand grid operators are supposed to invest in their grid infrastructure, implying expansion of power grid, information technology and measurement facilities. This is mainly due to the increasing amount of decentralized generation, resulting in dramatically changed demands on distribution grids which were originally planned for predictable and unidirectional power flow from the hand-over points to the end customers and to inferior grids. The high variability of load and generation - especially of feeders based on renewable sources - results in fast changing, hardly determinable and possibly illegal equipment utilization. In the future, even more DG sources will feed into distribution grids. These new challenges strictly necessitate intelligent energy management systems.

To enable energy management systems to find equitable operation strategies and to detect overstressing of lines and transformers, knowledge of grid state and equipment utilization is a matter of vital importance. Unfortunately, measurement technique is sparsely spread in distribution grids. Hence, grid state is not completely observable and has to be estimated. That is why research on state identification methods of underdetermined power systems is done. 


\section{Boundary Load Flow}

In general, underdetermined equation systems offer an infinite number of solutions, whereof in power systems only a part of them are actually possible. The boundary load flow method limits the solution space to these thinkable solutions by introducing constraints for all influencing variables. Thereby, the amount of solutions remains infinite, indeed. Unlike probabilistic power flow computation, power density functions and correlations are unknown.

So, in contrast to the other grid state identification methods the boundary load flow approach does not aim at finding one determined grid state. Instead, this method finds maxima and minima of state variables which actually are of less informative value than consequential resultant equipment utilization bounds. Thus, the latter is aspired.

Terminal currents are regarded as objectives due to continuous cable utilization is limited by currents. Nevertheless, dispersion of nodal powers is likewise valuable information and can be calculated in almost the same manner.

If drag indicators are installed at substation transformers to record maximum and minimum utilization, these values can be used as interval bounds. Otherwise current bounds can be obtained from transformer rating plates. In doing so, for each node upper and lower bounds of currents and powers are available. At switching stations all bounds are zero.

\subsection{Boundary Current Flow}

As mentioned above, for each node a current interval can be determined.

$$
I_{\mathrm{K}, v, \text { act }} \in\left[I_{\mathrm{K}, v, \text { min }}, I_{\mathrm{K}, v, \text { max }}\right]
$$

To calculate voltage alteration against nodal currents, Eq. (1) is reformulated

$$
\underline{\boldsymbol{Y}}_{\mathrm{KK}} \Delta \underline{\boldsymbol{u}}_{\mathrm{K}}=\Delta \underline{\boldsymbol{i}}_{\mathrm{K}}
$$

The slack voltage is independent of load situation, so $\Delta \underline{\boldsymbol{u}}_{\mathrm{K}, \mathrm{sl}}$ is always 0 . This constraint is inserted into Eq. (2) and replaces the former slack line of $\underline{\boldsymbol{Y}}_{\mathrm{KK}}$.

or shorter

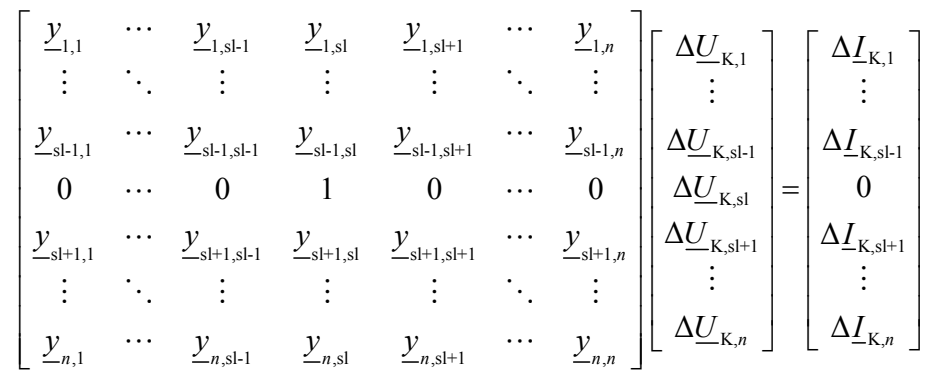

Inversion of Eq. (3) and insertion into the terminal voltage equation results in

$$
\Delta \underline{\boldsymbol{u}}_{\mathrm{T}}=\boldsymbol{K}_{\mathrm{KT}}^{\mathrm{T}} \underline{\boldsymbol{Y}}_{\mathrm{KK}, \mathrm{red}}^{-1} \Delta \underline{\boldsymbol{i}}_{\mathrm{K}, \mathrm{red}}
$$

Finally, Eq. (5) is inserted into the terminal current equation.

$$
\Delta \underline{\boldsymbol{i}}_{\mathrm{T}}=\underline{\boldsymbol{Y}}_{\mathrm{T}} \boldsymbol{K}_{\mathrm{KT}}^{\mathrm{T}} \underline{\boldsymbol{Y}}_{\mathrm{KK}, \text { red }}^{-1} \Delta \underline{\boldsymbol{i}}_{\mathrm{K}, \text { red }}
$$

or shorter 


$$
\Delta \underline{\boldsymbol{i}}_{\mathrm{T}}=\underline{\boldsymbol{D}}_{\mathrm{I}, \mathrm{I}} \Delta \underline{\boldsymbol{i}}_{\mathrm{K}, \mathrm{red}}
$$

with the current dispersion matrix $\underline{\boldsymbol{D}}_{\mathrm{I}, \mathrm{I}}$. Considering grids without capacitive shunts, $\underline{\boldsymbol{D}}_{\mathrm{I}, \mathrm{I}}$ is real-valued.

To find maximum and minimum of each coefficient of $\Delta \underline{\boldsymbol{i}}_{\mathrm{T}}$, Eq. (6) combined with the nodal current bounds formulates a continuous constrained linear optimization problem which can be solved by well known methods i.e. the Simplex-Algorithm.

\subsection{Boundary Power Flow}

Accordant to current bounds, maxima and minima of substation powers can be obtained from drag indicators or substation transformer rating plates. In the latter case

$$
-\boldsymbol{s}_{\mathrm{r}} \leq \boldsymbol{s}_{\mathrm{K}, \text { act }} \leq \boldsymbol{s}_{\mathrm{r}}
$$

Again, a linear dependency of nodal voltages on nodal powers is needed, but contrarily to boundary current flow calculation, power is a quadratic function against voltages.

$$
\underline{\boldsymbol{s}}_{\mathrm{K}}=3 \underline{\boldsymbol{U}}_{\mathrm{K}}\left(\underline{\boldsymbol{Y}}_{\mathrm{KK}} \underline{\boldsymbol{u}}_{\mathrm{K}}\right)^{*}
$$

Thus, accordant to power flow calculation, Eq. (9) is linearized by first-order Taylor-series expansion, such that

$$
\boldsymbol{J}\left[\begin{array}{c}
\Delta \boldsymbol{u}_{\mathrm{K}, \mathrm{r}} \\
\Delta \boldsymbol{u}_{\mathrm{K}, \mathrm{i}}
\end{array}\right]=\left[\begin{array}{cc}
\boldsymbol{J}_{\mathrm{A}} & \boldsymbol{J}_{\mathrm{B}} \\
\boldsymbol{J}_{\mathrm{C}} & \boldsymbol{J}_{\mathrm{D}}
\end{array}\right]\left[\begin{array}{c}
\Delta \boldsymbol{u}_{\mathrm{K}, \mathrm{r}} \\
\Delta \boldsymbol{u}_{\mathrm{K}, \mathrm{i}}
\end{array}\right]=\left[\begin{array}{c}
\Delta \boldsymbol{p} \\
-\Delta \boldsymbol{q}
\end{array}\right]
$$

Split-up into real and imaginary part doubles the equation system size, so two constraints for the slack node voltages have to be inserted into Eq. (10). $\boldsymbol{J}_{\mathrm{A}}$ and $\boldsymbol{J}_{\mathrm{D}}$ are modified in the same way as demonstrated in Eq. (3), whereas slack lines of $\boldsymbol{J}_{\mathrm{B}}$ and $\boldsymbol{J}_{\mathrm{C}}$ are replaced by zeros. Inversion now results in

$$
\left[\begin{array}{c}
\Delta \boldsymbol{u}_{\mathrm{K}, \mathrm{r}} \\
\Delta \boldsymbol{u}_{\mathrm{K}, \mathrm{i}}
\end{array}\right]=\boldsymbol{J}_{\text {red }}^{-1}\left[\begin{array}{c}
\Delta \boldsymbol{p} \\
-\Delta \boldsymbol{q}
\end{array}\right]=\left[\begin{array}{cc}
\boldsymbol{L}_{\mathrm{A}} & \boldsymbol{L}_{\mathrm{B}} \\
\boldsymbol{L}_{\mathrm{C}} & \boldsymbol{L}_{\mathrm{D}}
\end{array}\right]\left[\begin{array}{c}
\Delta \boldsymbol{p}_{\mathrm{red}} \\
-\Delta \boldsymbol{q}_{\mathrm{red}}
\end{array}\right]
$$

At the actual operation point, it is imperative that

$$
\begin{aligned}
& \boldsymbol{L}_{\mathrm{A}}=\boldsymbol{L}_{\mathrm{D}} \\
& \boldsymbol{L}_{\mathrm{B}}=-\boldsymbol{L}_{\mathrm{C}}
\end{aligned}
$$

The Jacobian of the last power flow iteration step offers a good approximation for $\boldsymbol{J}$ which virtually fulfills Eq. (12).

Due to Eq. (12) it is assumed that

such that

$$
\underline{\boldsymbol{L}}=\boldsymbol{L}_{\mathrm{A}}+\mathrm{j} \boldsymbol{L}_{\mathrm{B}}
$$

$$
\Delta \underline{\boldsymbol{u}}_{\mathrm{K}}=\underline{\boldsymbol{L}} \Delta \underline{\boldsymbol{s}}_{\mathrm{K}, \mathrm{red}}
$$

Following Eq. (5) and Eq. (6) a linear dependency of terminal currents on nodal powers is given

or shorter

$$
\Delta \underline{\boldsymbol{i}}_{\mathrm{T}}=\underline{\boldsymbol{Y}}_{\mathrm{T}} \boldsymbol{K}_{\mathrm{KT}}^{\mathrm{T}} \underline{\boldsymbol{L}} \Delta \underline{\boldsymbol{s}}_{\mathrm{K}, \mathrm{red}}
$$

with the power dispersion matrix $\underline{\boldsymbol{D}}_{\mathrm{I}, \mathrm{S}}$.

$$
\Delta \underline{\boldsymbol{i}}_{\mathrm{T}}=\underline{\boldsymbol{D}}_{\mathrm{I}, \mathrm{S}} \Delta \underline{\boldsymbol{s}}_{\mathrm{K}, \mathrm{red}}
$$


In combination with the nodal power constraints, Eq. (16) formulates a continuous constrained linear optimization problem which can be solved by well known methods, i.e. the Simplex-Algorithm, just like the boundary current flow.

\section{Sensitivity analysis}

Sensitivity analysis is used to systematically determine the effect of any arbitrary influencing variable on all state variables. Therefore, nonlinear system equations are linearized at a known operating point to achieve a linear dependency of state variables on influencing variables.

In the case of electric power systems, to simplify matters, typically complex nodal voltages are used as state variables whereas all measurable values can be considered as influencing variables, for example nodal powers, current magnitudes or even voltage magnitudes. Due to the required linear dependency a power flow calculation is not necessary.

'Known' operating points are system states in which all state variables and all influencing variables are known and consistent. They can be achieved by power flow calculation using a nodal power vector for example with all zero elements, rated powers of transformers or a presumption of load situation according to chapter 5 . The easiest and clearest way is the first one which is therefore used in the following sections.

\subsection{Sensitivity Matrices}

As mentioned above normally only information on magnitudes of currents, voltages and apparent powers is available. Solely at hand-over-points, plant farms or special customers active and reactive powers are measured separately and appropriately signed. Taking this into account sensitivity matrices for these three cases have to be developed.

With

$$
\boldsymbol{Y}_{\mathrm{KK}, \mathrm{r}}=\operatorname{Re}\left\{\underline{\boldsymbol{Y}}_{\mathrm{KK}}\right\}
$$

and

$$
\boldsymbol{Y}_{\mathrm{KK}, \mathrm{i}}=\operatorname{Im}\left\{\underline{\boldsymbol{Y}}_{\mathrm{KK}}\right\}
$$

The nodal current equation can be reformulated

or shorter

$$
\left[\begin{array}{cc}
\boldsymbol{Y}_{\mathrm{KK}, \mathrm{r}} & -\boldsymbol{Y}_{\mathrm{KK}, \mathrm{i}} \\
\boldsymbol{Y}_{\mathrm{KK}, \mathrm{i}} & \boldsymbol{Y}_{\mathrm{KK}, \mathrm{r}}
\end{array}\right]\left[\begin{array}{c}
\boldsymbol{u}_{\mathrm{K}, \mathrm{r}} \\
\boldsymbol{u}_{\mathrm{K}, \mathrm{i}}
\end{array}\right]=\left[\begin{array}{c}
\boldsymbol{i}_{\mathrm{K}, \mathrm{r}} \\
\boldsymbol{i}_{\mathrm{K}, \mathrm{i}}
\end{array}\right]=\left[\begin{array}{c}
\boldsymbol{I}_{\mathrm{K}} \cos \left(\boldsymbol{\varphi}_{\mathrm{K}}\right) \\
\boldsymbol{I}_{\mathrm{K}} \sin \left(\boldsymbol{\varphi}_{\mathrm{K}}\right)
\end{array}\right]
$$

Deletion of both slack lines results in

$$
\boldsymbol{Y}_{\mathrm{KK}, \mathrm{ext}} \boldsymbol{u}_{\mathrm{K}, \mathrm{ext}}=\boldsymbol{i}_{\mathrm{K}, \mathrm{ext}}
$$

$$
\boldsymbol{Y}_{\mathrm{KK}, \text { red }} \boldsymbol{u}_{\mathrm{K}, \text { red }}=\boldsymbol{i}_{\mathrm{K}, \text { red }}
$$

Inversion of Eq. (21) now leads to

$$
\left[\begin{array}{c}
\boldsymbol{u}_{\mathrm{K}, \text { red, } \mathrm{r}} \\
\boldsymbol{u}_{\mathrm{K}, \text { red, } \mathrm{i}}
\end{array}\right]=\boldsymbol{Y}_{\mathrm{KK}, \text { red }}^{-1} \boldsymbol{i}_{\mathrm{K}, \text { red }}=\boldsymbol{Z}_{\mathrm{KK}, \text { red }}\left[\begin{array}{c}
\boldsymbol{I}_{\mathrm{K}, \text { red }} \cos \left(\boldsymbol{\varphi}_{\mathrm{K}, \text { red }}\right) \\
\boldsymbol{I}_{\mathrm{K}, \text { red }} \sin \left(\boldsymbol{\varphi}_{\mathrm{K}, \text { red }}\right)
\end{array}\right]
$$

A linear dependency of $\boldsymbol{u}_{\mathrm{K}, \text { red }}$ on $\boldsymbol{i}_{\mathrm{K}, \text { red }}$ and $\boldsymbol{\varphi}_{\mathrm{K} \text {,red }}$ is achieved by first-order Taylor series expansion. 


$$
\boldsymbol{u}_{\mathrm{K}} \approx \boldsymbol{u}_{\mathrm{K}, 0}+\Delta \boldsymbol{u}_{\mathrm{K}}
$$

$\boldsymbol{u}_{\mathrm{K}, 0}$ is the known operation point voltage and $\Delta \boldsymbol{u}_{\mathrm{K}}$ the linearized deviation

$$
\left[\begin{array}{c}
\Delta \boldsymbol{u}_{\mathrm{K}, \mathrm{r}} \\
\Delta \boldsymbol{u}_{\mathrm{K}, \mathrm{i}}
\end{array}\right]=A_{\mathrm{UK}, \mathrm{IK}}\left[\begin{array}{c}
\Delta \varphi_{\mathrm{K}} \\
\Delta i_{\mathrm{K}}
\end{array}\right]
$$

with the Jacobian $\boldsymbol{A}_{\mathrm{UK}, \mathrm{IK}}$ which is already the requested sensitivity matrix

$$
\boldsymbol{A}_{\mathrm{UK}, \mathrm{IK}}=\frac{\partial\left(\boldsymbol{Z}_{\mathrm{KK}, \mathrm{red}}\left[\begin{array}{l}
\boldsymbol{I}_{\mathrm{K}, \mathrm{red}} \cos \left(\boldsymbol{\varphi}_{\mathrm{K}, \mathrm{red}}\right) \\
\boldsymbol{I}_{\mathrm{K}, \text { red }} \sin \left(\boldsymbol{\varphi}_{\mathrm{K}, \mathrm{red}}\right)
\end{array}\right]\right)}{\partial\left[\begin{array}{ll}
\boldsymbol{\varphi}_{\mathrm{K}}^{\mathrm{T}} & \boldsymbol{i}_{\mathrm{K}}^{\mathrm{T}}
\end{array}\right]}
$$

$\boldsymbol{A}_{\mathrm{UK}, \mathrm{IK}}$ can be separated into four sub matrices

$$
\boldsymbol{A}_{\mathrm{UK}, \mathrm{IK}}=\left[\begin{array}{ll}
\boldsymbol{A}_{\mathrm{Ur}, \varphi \mathrm{I}} & \boldsymbol{A}_{\mathrm{Ur}, \mathrm{I}} \\
\boldsymbol{A}_{\mathrm{Ui}, \varphi \mathrm{I}} & \boldsymbol{A}_{\mathrm{Ui}, \mathrm{I}}
\end{array}\right]
$$

Coefficients of all sub matrices can easily be calculated

$$
\begin{gathered}
\boldsymbol{A}_{\mathrm{Ur}, \varphi \mathrm{i}}=-\boldsymbol{Z}_{\mathrm{KK}, \mathrm{r}} \boldsymbol{I}_{\mathrm{K}, 0} \sin \left(\boldsymbol{\varphi}_{\mathrm{K}, 0}\right)-\boldsymbol{Z}_{\mathrm{KK}, \mathrm{i}} \boldsymbol{I}_{\mathrm{K}, 0} \cos \left(\boldsymbol{\varphi}_{\mathrm{K}, 0}\right) \\
\boldsymbol{A}_{\mathrm{Ur}, \mathrm{I}}=\boldsymbol{Z}_{\mathrm{KK}, \mathrm{r}} \cos \left(\boldsymbol{\varphi}_{\mathrm{K}, 0}\right)-\boldsymbol{Z}_{\mathrm{KK}, \mathrm{i}} \sin \left(\boldsymbol{\varphi}_{\mathrm{K}, 0}\right) \\
\boldsymbol{A}_{\mathrm{Ui}, \varphi \mathrm{i}}=-\boldsymbol{Z}_{\mathrm{KK}, \mathrm{i}} \boldsymbol{I}_{\mathrm{K}, 0} \sin \left(\boldsymbol{\varphi}_{\mathrm{K}, 0}\right)+\boldsymbol{Z}_{\mathrm{KK}, \mathrm{r}} \boldsymbol{I}_{\mathrm{K}, 0} \cos \left(\boldsymbol{\varphi}_{\mathrm{K}, 0}\right)
\end{gathered}
$$

and

$$
\boldsymbol{A}_{\mathrm{Ui, \textrm {I }}}=\boldsymbol{Z}_{\mathrm{KK}, \mathrm{i}} \cos \left(\varphi_{\mathrm{K}, 0}\right)+Z_{\mathrm{KK}, \mathrm{r}} \sin \left(\varphi_{\mathrm{K}, 0}\right)
$$

This leads to the following linear dependencies

$$
\Delta u_{\mathrm{K}, \mathrm{r}}=A_{\mathrm{Ur}, \varphi \mathrm{i}} \Delta \varphi_{\mathrm{K}}+A_{\mathrm{Ur}, \mathrm{I}} \Delta i_{\mathrm{K}}
$$

and

$$
\Delta \boldsymbol{u}_{\mathrm{K}, \mathrm{i}}=\boldsymbol{A}_{\mathrm{Ui}, \varphi \mathrm{i}} \Delta \boldsymbol{\varphi}_{\mathrm{K}}+\boldsymbol{A}_{\mathrm{Ui,}, \mathrm{I}} \Delta \boldsymbol{i}_{\mathrm{K}}
$$

If information on phase angles is not available, the first summands of Eq. (31) and Eq. (32) have to be omitted.

$$
\left[\begin{array}{c}
\Delta \boldsymbol{u}_{\mathrm{K}, \mathrm{r}} \\
\Delta \boldsymbol{u}_{\mathrm{K}, \mathrm{i}}
\end{array}\right]=\left[\begin{array}{c}
\boldsymbol{A}_{\mathrm{Ur}, \mathrm{I}} \\
\boldsymbol{A}_{\mathrm{Ui}, \mathrm{I}}
\end{array}\right] \Delta \boldsymbol{i}_{\mathrm{K}}
$$

Alternatively sensitivity matrices can be set up using polar coordinates. Therefore, the nodal current equation has to be reformulated into nonlinear equations of phase angles

$$
\left[\begin{array}{c}
\varphi_{\mathrm{K} 1} \\
\vdots \\
\varphi_{\mathrm{K} n}
\end{array}\right]=\boldsymbol{f}_{\varphi \mathrm{K}}\left(\left[\begin{array}{c}
\delta_{\mathrm{K} 1} \\
\vdots \\
\delta_{\mathrm{K} n}
\end{array}\right],\left[\begin{array}{c}
U_{\mathrm{K} 1} \\
\vdots \\
U_{\mathrm{K} n}
\end{array}\right]\right)
$$

and magnitudes

$$
\left[\begin{array}{c}
I_{\mathrm{K} 1} \\
\vdots \\
I_{\mathrm{K} n}
\end{array}\right]=\boldsymbol{f}_{\mathrm{IK}}\left(\left[\begin{array}{c}
\delta_{\mathrm{K} 1} \\
\vdots \\
\delta_{\mathrm{K} n}
\end{array}\right],\left[\begin{array}{c}
U_{\mathrm{K} 1} \\
\vdots \\
U_{\mathrm{K} n}
\end{array}\right]\right)
$$

First-order Taylor series expansion around the operation point results in 


$$
\left[\begin{array}{c}
\varphi_{\mathrm{K} 1} \\
\vdots \\
\varphi_{\mathrm{K} n} \\
\hdashline I_{\mathrm{K} 1} \\
\vdots \\
I_{\mathrm{K} n}
\end{array}\right] \approx\left[\begin{array}{c}
\varphi_{\mathrm{K} 1,0} \\
\vdots \\
\varphi_{\mathrm{K} n, 0} \\
I_{\mathrm{K} 1,0} \\
\vdots \\
I_{\mathrm{K} n, 0}
\end{array}\right]+\left[\begin{array}{c}
\Delta \varphi_{\mathrm{K} 1} \\
\vdots \\
\Delta \varphi_{\mathrm{K} n} \\
\hdashline \Delta I_{\mathrm{K} 1} \\
\vdots \\
\Delta I_{\mathrm{K} n}
\end{array}\right]
$$

with

and the Jacobian

$$
\left[\begin{array}{c}
\Delta \varphi_{\mathrm{K} 1} \\
\vdots \\
\Delta \varphi_{\mathrm{K} n} \\
\hline \Delta I_{\mathrm{K} 1} \\
\vdots \\
\Delta I_{\mathrm{K} n}
\end{array}\right]=\left[\frac{\partial \boldsymbol{f}_{\varphi}}{\partial[\boldsymbol{\delta} \mid \boldsymbol{u}]^{\mathrm{T}}}\left[\begin{array}{c}
\Delta \delta_{\mathrm{K} 1} \\
\vdots \\
\left.\frac{\partial \boldsymbol{f}_{\mathrm{I}}}{\partial[\boldsymbol{\delta}} \boldsymbol{u}\right]^{\mathrm{T}}
\end{array}\right]\left[\begin{array}{c}
\Delta \delta_{\mathrm{K} n} \\
\Delta U_{\mathrm{K} 1} \\
\vdots \\
\Delta U_{\mathrm{K} n}
\end{array}\right]\right.
$$

which is set up as shown in Eq. (39)

$$
\boldsymbol{J}_{\mathrm{I}}=\left[\frac{\frac{\partial \boldsymbol{f}_{\varphi}}{\partial[\boldsymbol{\delta}: \boldsymbol{u}]^{\mathrm{T}}}}{\hdashline \frac{\partial \boldsymbol{f}_{\mathrm{I}}}{\partial[\boldsymbol{\delta}: \boldsymbol{u}]^{\mathrm{T}}}}\right]
$$

$$
\boldsymbol{J}_{\mathrm{I}}=\left[\begin{array}{ccc:ccc}
\frac{\partial \varphi_{\mathrm{K} 1}}{\partial \delta_{\mathrm{K} 1}} & \cdots & \frac{\partial \varphi_{\mathrm{K} 1}}{\partial \delta_{\mathrm{K} n}} & \frac{\partial \varphi_{\mathrm{K} 1}}{\partial U_{\mathrm{K} 1}} & \cdots & \frac{\partial \varphi_{\mathrm{K} 1}}{\partial U_{\mathrm{K} n}} \\
\vdots & \ddots & \vdots & \vdots & \ddots & \vdots \\
\frac{\partial \varphi_{\mathrm{K} n}}{\partial \delta_{\mathrm{K} 1}} & \cdots & \frac{\partial \varphi_{\mathrm{K} n}}{\partial \delta_{\mathrm{K} n}} & \frac{\partial \varphi_{\mathrm{K} n}}{\partial U_{\mathrm{K} 1}} & \cdots & \frac{\partial \varphi_{\mathrm{K} n}}{\partial U_{\mathrm{K} n}} \\
\hdashline \frac{\partial I_{\mathrm{K} 1}}{\partial \delta_{\mathrm{K} 1}} & \cdots & \frac{\partial I_{\mathrm{K} 1}}{\partial \delta_{\mathrm{K} n}} & \frac{\partial I_{\mathrm{K} 1}}{\partial U_{\mathrm{K} 1}} & \cdots & \frac{\partial I_{\mathrm{K} 1}}{\partial U_{\mathrm{K} n}} \\
\vdots & \ddots & \vdots & \vdots & \ddots & \vdots \\
\frac{\partial I_{\mathrm{K} n}}{\partial \delta_{\mathrm{K} 1}} & \cdots & \frac{\partial I_{\mathrm{K} n}}{\partial \delta_{\mathrm{K} n}} & \frac{\partial I_{\mathrm{K} n}}{\partial U_{\mathrm{K} 1}} & \cdots & \frac{\partial I_{\mathrm{K} n}}{\partial U_{\mathrm{K} n}}
\end{array}\right]
$$

After removing both slack lines in Eq. (39), inversion leads to

$$
\boldsymbol{B}_{\mathrm{UK}, \mathrm{IK}}=\boldsymbol{J}_{\mathrm{I}, \mathrm{red}}^{-1}=\left[\begin{array}{c:c}
\boldsymbol{B}_{\delta, \varphi} & \boldsymbol{B}_{\delta, \mathrm{I}} \\
\hdashline \boldsymbol{B}_{\mathrm{U}, \varphi} & \boldsymbol{B}_{\mathrm{U}, \mathrm{I}}
\end{array}\right]
$$

Phase angles and magnitudes of nodal voltages can now be approximated.

and

$$
\left[\begin{array}{c}
\Delta \delta_{\mathrm{K} 1} \\
\vdots \\
\Delta \delta_{\mathrm{K} n}
\end{array}\right]=\boldsymbol{B}_{\delta, \varphi}\left[\begin{array}{c}
\Delta \varphi_{\mathrm{K} 1} \\
\vdots \\
\Delta \varphi_{\mathrm{K} n}
\end{array}\right]+\boldsymbol{B}_{\delta, \mathrm{I}}\left[\begin{array}{c}
\Delta I_{\mathrm{K} 1} \\
\vdots \\
\Delta I_{\mathrm{K} n}
\end{array}\right]
$$




$$
\left[\begin{array}{c}
\Delta U_{\mathrm{K} 1} \\
\vdots \\
\Delta U_{\mathrm{K} n}
\end{array}\right]=\boldsymbol{B}_{\mathrm{U}, \varphi}\left[\begin{array}{c}
\Delta \varphi_{\mathrm{K} 1} \\
\vdots \\
\Delta \varphi_{\mathrm{K} n}
\end{array}\right]+\boldsymbol{B}_{\mathrm{U}, \mathrm{I}}\left[\begin{array}{c}
\Delta I_{\mathrm{K} 1} \\
\vdots \\
\Delta I_{\mathrm{K} n}
\end{array}\right]
$$

Due to the extensive nonlinearity of square-root and arc tangent functions, the approximation error is expected to be greater compared to Cartesian approximation. Again, if information on phase angles is not available, the first summand has to be omitted in Eq. (41) and Eq. (42) which leads to additional approximation errors.

Calculation of apparent power sensitivity matrices is similar and sensitivity matrices based on active and reactive power can calculated in terms of the power flow Jacobian.

\subsection{Terminal values}

In addition to nodal values, terminal measurands may be used for sensitivity analysis as well. Calculation of terminal-based sensitivity matrices proves to be more difficult due to the obtained Jacobians, which are set up analogously to nodal measurand matrices, are not square and thus not invertible. Sole exception is a single branch line with two nodes. So as a rule, grids consist of more terminals than nodes and thus the initial equation system is underdetermined. One reasonable solution approach out of an infinite number is the calculation of pseudo-inverses according to the method of Moore-Penrose.

For each influencing variable $g$ starting with the initial equation

$$
\Delta \boldsymbol{g}=\boldsymbol{J} \Delta \boldsymbol{u}
$$

the method of Moore-Penrose generates a matrix $\boldsymbol{M}$ with the dimension of $\boldsymbol{J}^{\mathrm{T}}$ such that

$$
\boldsymbol{M} \Delta \boldsymbol{g}=\Delta \boldsymbol{u}
$$

$\boldsymbol{M}$ has to satisfy the constraints

$$
\begin{aligned}
& \boldsymbol{J} \boldsymbol{M} \boldsymbol{J}=\boldsymbol{J} \\
& \boldsymbol{M} \boldsymbol{J} \boldsymbol{M}=\boldsymbol{M} \\
& \boldsymbol{J} \boldsymbol{M}=(\boldsymbol{J} \boldsymbol{M})^{* \mathrm{~T}} \\
& \boldsymbol{M} \boldsymbol{J}=(\boldsymbol{M} \boldsymbol{J})^{* \mathrm{~T}}
\end{aligned}
$$

and particularly stands out from all other possible solutions due to

$$
\|\Delta u\|=\|\boldsymbol{M} \Delta \boldsymbol{g}\|
$$

is minimized.

In general, due to equivocality terminal measurands are rather ineligible for grid state identification based on sensitivity analysis. Furthermore, simulations proved that approximation error is vastly higher compared to the approach solely based on nodal values. Thus, in the following chapters nodal measurands are focused. Nevertheless, the algorithm works analogously using terminal values. If selected nodal and terminal values are not interdependent, it is even possible to combine them and use both for state identification, although in the majority of cases interdependency is given.

\subsection{State identification}

Provided that at each possible measuring location at the most one device is installed and only magnitudes are recorded, a measuring vector $\boldsymbol{m}$ of dimension $4 k \times 1$ can be introduced 


$$
\boldsymbol{m}=\left[\begin{array}{c}
\boldsymbol{m}_{\mathrm{iK}} \\
\boldsymbol{m}_{\mathrm{sK}} \\
\boldsymbol{m}_{\mathrm{pK}} \\
\boldsymbol{m}_{\mathrm{qK}}
\end{array}\right]
$$

Each measuring device can either record

- $\quad$ magnitude and phase angle or solely magnitude of current

- $\quad$ magnitude and phase angle or solely magnitude of apparent power

- $\quad$ active or reactive power or both active and reactive power

Sub vectors of $\boldsymbol{m}$ contain measured values at the corresponding positions. Residual elements are either filled with predicted values or are set to zero. Sequence of sub vectors is irrelevant and if possibly phase angles are measured, $\boldsymbol{m}$ has to be accordingly extended.

Depending on the sequence of sub vectors, all possible sensitivity matrices can be combined to

$$
\left[\begin{array}{c}
\Delta \boldsymbol{u}_{\mathrm{K}, \mathrm{r}} \\
\Delta \boldsymbol{u}_{\mathrm{K}, \mathrm{i}}
\end{array}\right]=\boldsymbol{A} \boldsymbol{m}
$$

with the sensitivity matrix

$$
\boldsymbol{A}=\left[\begin{array}{llll}
\boldsymbol{A}_{\mathrm{Ur}, \mathrm{I}} & \boldsymbol{A}_{\mathrm{Ur}, \mathrm{S}} & \boldsymbol{A}_{\mathrm{Ur}, \mathrm{P}} & \boldsymbol{A}_{\mathrm{Ur}, \mathrm{Q}} \\
\boldsymbol{A}_{\mathrm{Ui}, \mathrm{I}} & \boldsymbol{A}_{\mathrm{Ui}, \mathrm{S}} & \boldsymbol{A}_{\mathrm{Ui}, \mathrm{P}} & \boldsymbol{A}_{\mathrm{Ui}, \mathrm{Q}}
\end{array}\right]
$$

Alternatively in polar coordinates

$$
\left[\begin{array}{c}
\Delta \boldsymbol{\delta}_{\mathrm{K}} \\
\Delta \boldsymbol{u}_{\mathrm{K}}
\end{array}\right]=\boldsymbol{B} \boldsymbol{m}
$$

with

$$
\boldsymbol{B}=\left[\begin{array}{cccc}
\boldsymbol{B}_{\delta, \mathrm{I}} & \boldsymbol{B}_{\delta, \mathrm{S}} & \boldsymbol{B}_{\delta, \mathrm{P}} & \boldsymbol{B}_{\delta, \mathrm{Q}} \\
\boldsymbol{B}_{\mathrm{U}, \mathrm{I}} & \boldsymbol{B}_{\mathrm{U}, \mathrm{S}} & \boldsymbol{B}_{\mathrm{U}, \mathrm{P}} & \boldsymbol{B}_{\mathrm{U}, \mathrm{Q}}
\end{array}\right]
$$

The grid state can now be estimated using the developed linearized models by determining the deviation from the known operation point. As mentioned above, in this work the idling grid is used as operation point due to the entire consistent and complex-valued grid state can be easily obtained and no modifications of the measured values are needed.

In Cartesian coordinates the grid state is estimated as follows

$$
\left[\begin{array}{c}
\boldsymbol{u}_{\mathrm{K}, \mathrm{r}, \mathrm{est}} \\
\boldsymbol{u}_{\mathrm{K}, \mathrm{i}, \mathrm{est}}
\end{array}\right]=\left[\begin{array}{c}
\boldsymbol{u}_{\mathrm{K}, \mathrm{r}, 0} \\
\boldsymbol{u}_{\mathrm{K}, \mathrm{i}, 0}
\end{array}\right]+\boldsymbol{A} \boldsymbol{m}
$$

and in polar coordinates respectively

$$
\left[\begin{array}{l}
\boldsymbol{\delta}_{\mathrm{K}, \text { est }} \\
\boldsymbol{u}_{\mathrm{K}, \text { est }}
\end{array}\right]=\left[\begin{array}{l}
\boldsymbol{\delta}_{\mathrm{K}, 0} \\
\boldsymbol{u}_{\mathrm{K}, 0}
\end{array}\right]+\boldsymbol{B} \boldsymbol{m}
$$

At the operation point, the difference between actual and estimated grid state is zero. The estimation error increases with growing deviation from the operation point. Thus, error estimation should be applied. 


\section{Nodal Load Decomposition}

Grid state identification algorithms depend on some information on grid condition. In low and medium voltage grids this information is not available in sufficient amount to calculate a unique grid state. One possibility to bypass lack of information is to supplement available measurands by assumptions.

Further literature offer miscellaneous methods to forecast power time-series over a various amount of time. For the purpose of grid state identification a forecasting method is needed which solely gets by on historic and present measured time-series and information on grid topology. So it is totally satisfactory to roughly assume nodal voltage values. Therefore, measured time series are used to back-reference on load type mixture. With this additional information forecasting of nodal active power is possible using analytic load profiles. To predict reactive power as well, corresponding time-series are introduced.

\subsection{Active power profiles}

To simplify estimation of energy consumption of costumers within one year, the VDEW announced eleven different analytic load profiles merged into groups of domestic, industrial and agricultural type:

- $\quad$ common industry

- $\quad$ industry on workdays (8 am to $6 \mathrm{pm})$

- $\quad$ industry on workdays (evening)

- continuous industry

- $\quad$ stores and barbers

- bakeries

- $\quad$ industry on weekends

- $\quad$ common domestic

- common agriculture

- milk production

- miscellaneous agriculture

These profiles are obtained by averaging over a huge amount of customers of the same type within several years and do not represent the stochastic behavior of one single load. The eleven linear independent load profiles are supplemented by profiles describing off-peak storage heating, street lighting, heat pumps and other detachable loads.

\subsection{Nodal load decomposition}

The year is split up into three seasons: 'winter', 'transition' and 'summer'. Each season consists of three reference days: 'Workday', 'Saturday' and 'Sunday'. For each load type and for the reference days of all seasons, the VDEW announced a set of 96 values to be assigned to any quarter of an hour of that specific day, forming 99 unique daily time-series. Depending on the year, the federal state and the holidays they can be merged to 11 yearly time-series, one for each load type, and are normalized to $1000 \frac{\mathrm{kWh}}{\mathrm{a}}$.

Nodal load extraction offers the possibility of determining load composition from measured active power time-series of nodes or hand-over points. Therefore, a measured active power time series $\boldsymbol{p}_{\text {meas }}$ of the point of interest has to be available and should provide information 
on consumption of at least one year. Furthermore, the measuring time period and the set of $\mu$ load profiles have to be known. This set can contain all analytic load profiles as well as off-peak heating, street lighting and other specific or detachable load profiles. Additionally, it is possible to add some series of measured loads or feeders to the set to optimize extraction quality.

For each 35040 quarters of an hour within one year it is assumed that the resulting active power can be described as a sum of the corresponding values of all load types

$$
P(d, t)=\sum_{s=1}^{3} w_{s}(t) \sum_{p=1}^{\mu} P_{d, s, p}(t \bmod 96) w_{p}
$$

$d$ indicates the day type workday, Saturday or Sunday. $t$ is the time which has elapsed since $1^{\text {st }}$ of January, 0:00 o' clock in quarters of hours. Thus, within a year $t$ ranges from 0 to 35039 or 0 to 35135 in leap years, respectively. $w_{s}$ is a seasonal interpolation factor indicating the affiliation of $t$ to the seasons. This might be a block function clearly assigning each time step to one season. Better results are achieved using fading crossovers. All interpolation methods, i.e. splines, are suitable as long as

$$
\sum_{s=1}^{3} w_{s}(t)=1
$$

for all time steps. Furthermore, in Eq. (54) $w_{p}$ is the profile weighting vector indicating how many of each normalized profile types are connected to the node.

Due to the linear dependency of $P(t)$ on $w_{p}$ Eq. (54) can be written in matrix notation

$$
\boldsymbol{p}_{\text {cal }}=\boldsymbol{L} \boldsymbol{w}_{\mathrm{p}}
$$

To find the best fitting profile weighting vector $\boldsymbol{w}_{\mathrm{p}}$ for a measured time-series of active power, the difference between $\boldsymbol{p}_{\text {cal }}$ and $\boldsymbol{p}_{\text {meas }}$ has to be minimized.

$$
\min _{w_{\mathrm{p}}}(\Delta \boldsymbol{p})=\min _{w_{\mathrm{p}}}\left(\boldsymbol{L} \boldsymbol{w}_{\mathrm{p}}-\boldsymbol{p}_{\text {meas }}\right)
$$

Due to Eq. (57) is overdetermined results are obtained by linear least-squares optimization. Thus, $\boldsymbol{w}_{\mathrm{p}}$ can be directly calculated.

$$
\boldsymbol{w}_{\mathrm{p}}=\left(\boldsymbol{L}^{\mathrm{T}} \boldsymbol{L}\right)^{-1} \boldsymbol{L}^{\mathrm{T}} \boldsymbol{p}_{\text {meas }}
$$

Extraction quality decreases with increasing amount of fluctuating feeders which are hardly to forecast. It can be improved by out-counting weather-dependent feeders like wind turbines before load extraction which mostly is possible due to data availability. Alternatively, measuring period should be extended or average time series of several years should be used.

Besides, weighting shifts among agricultural load profiles might be possible due to similarity to themselves. For the same reason influence of this shifting on the results is almost negligible.

\subsection{Determination of Reactive Power Profiles}

Chronological sequence admits the presumption that customers exhibit not only a distinct time-series of active power but also of reactive power depending on time and weekday. Thus, it is reasonable to assume that reactive power as well can be expressed as a sum of load profiles at any discrete time interval. 


$$
Q(d, t)=\sum_{s=1}^{3} w_{s}(t) \sum_{p=1}^{\mu} Q_{d, s, p}(t \bmod 96) w_{p}
$$

In Eq. (59) $d, t, w_{p}$ and $w_{s}$ are the same as before. For each node $w_{p}$ is obtained by load type mixture extraction. So, in this case reactive power profiles $\boldsymbol{Q}_{\mathrm{d}, \mathrm{s}, \mathrm{p}}$ are unknown. $Q(d, t)$ is the measured reactive power time-series.

Due to linear dependency of $Q(d, t)$ on $\boldsymbol{Q}_{\mathrm{s}, \mathrm{p}}$ it is possible to reorder Eq. (59) in the style of Eq. (56). This leads to a linear equation system of 9504 unknowns.

$$
\boldsymbol{q}_{\text {meas }}=\boldsymbol{W}_{\mathrm{s}, \mathrm{p}} \boldsymbol{q}_{\mathrm{p}}
$$

Coefficients of $\boldsymbol{Q}_{\mathrm{s}, \mathrm{p}}$ are now found in vector $\boldsymbol{q}_{\mathrm{p}} \cdot \boldsymbol{W}_{\mathrm{s}, \mathrm{p}}$ is a sparse matrix containing the elements of $w_{s}(t)$. Seasonal weighting factors are linear dependent, so for each node

$$
\operatorname{rank}\left(\boldsymbol{W}_{\mathrm{s}, \mathrm{p}}\right) \leq 96 \cdot 3 \cdot 3=864<9504
$$

Thus, the resulting equation system is underdetermined. Furthermore, in addition to some unavoidable outage of measurement and to compensate statistical spread at least $\mu$ measured yearly times-series of different nodes with linear independent load type mixture are needed. To obtain reliable results utilization of more that $2 \mu$ time series is reasonable. In case exactly $2 \mu$ yearly time-series are considered, the equation system to be solved is

$$
\left[\begin{array}{c}
\boldsymbol{q}_{\text {meas }, 1} \\
\vdots \\
\boldsymbol{q}_{\text {meas }, 2 \mu}
\end{array}\right]=\left[\begin{array}{c}
\boldsymbol{W}_{\mathrm{s}, \mathrm{p}, 1} \\
\vdots \\
\boldsymbol{W}_{\mathrm{s}, \mathrm{p}, 2 \mu}
\end{array}\right] \boldsymbol{q}_{\mathrm{p}}
$$

Contrarily to active power, the contribution of capacitive shunts to the reactive power at the hand-over point is not negligible and has to be considered. Using an equipment model with concentrated parameters, for each node the contribution of connected capacitive shunts can be calculated

$$
Q_{\mathrm{K}, \mathrm{C}}=3 \omega C_{\mathrm{K}} U_{\mathrm{K}}^{2}
$$

where $C_{\mathrm{K}}$ is the sum of all capacitors attached to the node. Voltages are virtually never known in distribution grids. To anyway obtain binding approximations of time-series it is assumed that the capacitive contribution is practically constant due to the voltage deviation from nominal rating is less than $10 \%$. Thus, the overall contribution of capacitive shunts $Q_{\mathrm{C}}$ can be estimated.

$$
Q_{\mathrm{C}} \approx 3 \omega \sum_{i=1}^{\mathrm{k}} C_{\mathrm{K}, i} U_{\mathrm{nN}}^{2}
$$

Due to $Q_{\mathrm{C}}$ is treated as base load, this results in an offset of the aspired load profiles on the ordinate. In different grids this offset is unknown and has to be calculated prior to the state identification process by least-squares analysis. Furthermore, current-dependent contribution of inductive series-elements is on the one hand small and on the other hand hardly determinable in underdetermined grids. Hence it is ignored.

In the following figures reactive power time-series for the previously mentioned reference days for the domestic, an industrial and an agricultural VDEW load type are shown. They are obtained using 52 measured yearly time-series of hand-over point active and reactive powers out of 2006 and 2007. This results in an equation system of dimension 
$1.82 \cdot 10^{6} \times 9504$. After solving the least-squares problem a polynomial smoothing is applied using a $12^{\text {th }}$ order polynomial.

Relating reactive power profiles i.e. to $1000 \mathrm{kVArh}$ is not reasonable due to this would result in loss of load amount coherence.

Although no starting vector is given and no information on active power profiles is used during calculation, particularly the domestic and the industrial reactive power profiles strongly resembles the accordant VDEW profiles which can be seen as validation of the obtained results.

\subsection{State identification}

To obtain a complete power vector $\underline{\boldsymbol{s}}_{\mathrm{in}}$, the measured nodal powers $\underline{\boldsymbol{s}}_{\text {meas }}$ are supplemented by estimated nodal powers $\underline{\boldsymbol{s}}_{\text {est }}$. For each unobserved node active and reactive powers are forecasted.

$$
\underline{S}_{\text {est }}(t)=\sum_{s=1}^{3} w_{s}(t)\left(\sum_{p=1}^{\mu} P_{d, s, p}(t \bmod 96)+\mathrm{j} \sum_{p=1}^{\mu} Q_{d, s, p}(t \bmod 96)\right) w_{p}+\mathrm{j} Q_{\text {offset }}
$$

$Q_{\text {offset }}$ is calculated accordant to Eq. (58)

$$
Q_{\text {offset }}=\left(\boldsymbol{o}^{\mathrm{T}} \boldsymbol{o}\right)^{-1} \boldsymbol{o}^{\mathrm{T}}\left(\boldsymbol{Q}_{\text {meas }}-\sum \boldsymbol{Q}_{\text {profiles }}\right)
$$

with

$$
\boldsymbol{o}=\frac{1}{\sum C_{\mathrm{K}}}\left[\begin{array}{c}
C_{\mathrm{K}, 1} \\
\vdots \\
C_{\mathrm{K}, n}
\end{array}\right]
$$

and proportionally distributed to the nodes

$$
\boldsymbol{q}_{\text {offset }}=\boldsymbol{o} Q_{\text {offset }}
$$

$\underline{\boldsymbol{s}}_{\text {meas }}$ and $\underline{\boldsymbol{s}}_{\text {est }}$ are merged to $\underline{\boldsymbol{s}}_{\text {in }}$ which is used for a power flow calculation. The resulting nodal voltages determine the aspired grid state.

\section{Grid reduction approach}

The goal of grid state identification is to find the best estimation of grid condition using as few additional measurands as possible. Therefore sensitivity analysis targets on searching the most valuable measurands to keep estimation error within a predefined tolerance and investment cost on an acceptable ratio. On the other hand nodal load assumptions can be used to complete the underdetermined input vector, so the grid state can easily be estimated by power flow calculation. In contrast, grid reduction tries to more intensely exploit existent measurands particularly with regard to terminal values due to this information is almost discounted by sensitivity analysis. Therefore grid reduction offers a way to reconstruct lost information on voltage and current angles at bus bars. It is even possible that there is no need of installing new devices.

Typically only magnitudes of voltages at central busbars and current magnitudes of connected lines are measured. By reducing the grid to these central busbars and some auxiliary nodes the entire complex-valued state of the reduced grid can be estimated. These results offer inference on condition of the whole original grid. On the one hand they can be 
used for other grid state identification methods like sensitivity analysis and on the other hand they set the fundament for a method to recursively back-reference to the original grid state.

State identification by grid reduction is done by five consecutive steps which are shown in the following subchapters.

\subsection{Deletion of unobserved branch lines}

The first step is to eliminate branch lines with unobserved nodes at both ends such that the whole grid now only contains tie-lines starting and ending at observed bus bars. Nominal powers or currents of removed nodes are added to the remaining node on the tie-line the branch previously was connected to. This results in a new grid topology $\boldsymbol{K}_{\mathrm{KT} \text {,simp }}, \underline{\boldsymbol{Y}}_{\mathrm{T} \text {,simp }}$ and $\underline{\boldsymbol{Y}}_{\mathrm{KK}, \text { simp }}$ and new nodal and terminal influencing vectors

$$
\begin{aligned}
& \underline{\boldsymbol{g}}_{\mathrm{K}, \text { simp }}=\boldsymbol{K}_{\mathrm{K}, \text { simp,org }} \underline{\boldsymbol{g}}_{\mathrm{K}, \text { org }} \\
& \underline{\boldsymbol{g}}_{\mathrm{T}, \text { simp }}=\boldsymbol{K}_{\mathrm{T}, \text { simp,org }} \underline{\boldsymbol{g}}_{\mathrm{T}, \text { org }}
\end{aligned}
$$

where $\underline{g}$ universally represents power or current measurands.

Indeed, doing so results in a little error due to grid losses on the removed branch line are ignored, but this error is negligible. Nevertheless, maximum estimation errors will be encountered at unobserved branch lines during back-referencing due to missing information.

\subsection{Grid reduction}

In the new grid all tie-lines start and end at observed central switching stations but contain a varying number of unobserved nodes. In the next step all these nodes on each tie-line are removed and replaced by a single auxiliary node at a certain position on each line which carries the whole load of all removed nodes.

This method differs from conventional grid simplification methods at which the first and the last unobserved node of each line are used as auxiliary nodes and the rest of the tie-line is removed. The cumulated load is distributed to these nodes such that the inner lines are idle. Thus, grid losses can not be considered. Furthermore, due to missing information, a uniquely determinable power distribution is impossible using the mentioned previous approach.

Only tie-lines with more than one unobserved node have to be processed in this step. To obtain the position of the auxiliary node, first of all the parameters $r_{v}, x_{v}, c_{v}, g_{v}$ and the length $l_{v}$ of each complete tie-line $v$ between the observed nodes have to be calculated from the parameters of its $\mu$ subsections.

$$
r_{v}=\frac{\sum_{\mu}\left(r_{v, \mu} l_{v, \mu}\right)}{l_{v}}, \quad x_{v}=\frac{\sum_{\mu}\left(x_{v, \mu} l_{v, \mu}\right)}{l_{v}}, \quad c_{v}=\frac{\sum_{\mu}\left(c_{v, \mu} l_{v, \mu}\right)}{l_{v}}, \quad g_{v}=\frac{\sum_{\mu}\left(g_{v, \mu} l_{v, \mu}\right)}{l_{v}}
$$

and

$$
l_{v}=\sum_{\mu} l_{v, \mu}
$$

Each processed tie-line can be described as follows 


$$
\boldsymbol{K}_{\mathrm{K}, \mathrm{T}, V}=\left[\begin{array}{llll}
1 & 0 & 0 & 0 \\
0 & 1 & 1 & 0 \\
0 & 0 & 0 & 1
\end{array}\right]
$$

and

$$
\underline{\boldsymbol{Y}}_{\mathrm{T}, v}=\left[\begin{array}{cc:cc}
\underline{y}_{v, \text { left,aa }} & \underline{y}_{v, \text { left, ab }} & 0 & 0 \\
\underline{y}_{v, \text { left,ba }} & \underline{y}_{v, \text { left, bb }} & 0 & 0 \\
\hdashline 0 & 0 & \underline{y}_{v, \text { right, aa }} & \underline{y}_{v, \text { right, ab }} \\
0 & 0 & \underline{y}_{v, \text { right, ba }} & \underline{y}_{v, \text { right }, \mathrm{bb}}
\end{array}\right]
$$

For ease of better understanding it is assumed that the left line is connected to the node with the smaller number and the right line to the node with the higher number, respectively. The auxiliary node is placed between left and right line.

This method needs assumptions of active and reactive power of each unobserved node and thus depends on estimation quality. A rough estimation can be obtained by substation transformer rating plates. On the other hand, forecasted values offer a better approximation. Due to the fact that nodal voltages and terminal powers at the ends of each tie-line are known, the system is decoupled and all tie-lines can be calculated separately. Thus, in addition to the forecasted values at each unobserved node, it is assumed, that nodal powers of the observed nodes equal the negative value of the connected observed terminal. Now, the rule of torque can be applied.

$$
l_{v, \text { left }}=\frac{\sum_{\mu}\left(S_{v, \mu} d_{v, \mu}\right)}{\sum_{\mu} S_{v, \mu}}
$$

Analogously to the definition of left and right line, it is assumed that $d_{v, \mu}$ is the distance to the node with the smaller number. The parameters of the auxiliary lines are calculated as described above.

The decoupled tie-lines are now reassembled to the reduced grid which is described by $\boldsymbol{K}_{\mathrm{KT}, \text { red }}, \underline{\boldsymbol{Y}}_{\mathrm{T}, \mathrm{red}}$ and $\underline{\boldsymbol{Y}}_{\mathrm{KK}, \text { red }}$. For the purpose of better overview, nodes are renumbered such that

$$
\boldsymbol{K}_{\mathrm{KT}, \text { red }}=\left[\begin{array}{c}
\boldsymbol{K}_{\mathrm{KT}, \text { red,obs }} \\
\boldsymbol{K}_{\mathrm{KT}, \text { red,unobs }}
\end{array}\right]
$$

The reduced grid exhibits the following properties:

- no unobserved branches,

- a maximum of one unobserved node at each tie-line,

- $\quad$ all tie-lines start and end at observed nodes.

\subsection{Calcuation of the reduced grid state}

In the reduced grid unknown values which might be used as state variables are:

- $\quad$ voltage magnitudes at auxiliary nodes,

- all voltage angles except at the slack node,

- terminal current magnitudes connected to auxiliary nodes,

- $\quad$ all terminal current phase angles without exception. 
Nodal currents at auxiliary nodes are not used as state variable since they depend on terminal currents

$$
\underline{\boldsymbol{i}}_{\mathrm{K}, \text { unobs }}=\boldsymbol{K}_{\mathrm{KT} \text {,unobs }} \underline{\boldsymbol{i}}_{\mathrm{T}, \text { unobs }}
$$

It is obvious that the above mentioned variables can be divided into two groups: nodal and terminal values. Both groups offer the ability to uniquely describe the grid state. Therefore, two state vectors containing the unknown values are introduced.

$$
\boldsymbol{x}_{\text {red }}=\left[\begin{array}{c}
\boldsymbol{u}_{\mathrm{K}, \text { unobs }} \\
\boldsymbol{\delta}_{\mathrm{K}, \text { unobs }}
\end{array}\right]
$$

and

$$
\boldsymbol{y}_{\text {red }}=\left[\begin{array}{c}
\boldsymbol{i}_{\mathrm{T}, \text { unobs }} \\
\boldsymbol{\varphi}_{\mathrm{T}, \text { unobs }}
\end{array}\right]
$$

They are completed by the measured values such that

$$
\boldsymbol{x}_{\mathrm{u}}=\left[\begin{array}{c}
\boldsymbol{u}_{\mathrm{obs}} \\
\boldsymbol{u}_{\mathrm{K}, \text { unobs }}
\end{array}\right], \quad \boldsymbol{x}_{\delta}=\left[\begin{array}{c}
0 \\
\boldsymbol{\delta}_{\mathrm{K}, \text { unobs }}
\end{array}\right]
$$

and

$$
\boldsymbol{y}_{\mathrm{i}}=\left[\begin{array}{c}
\boldsymbol{i}_{\mathrm{obs}} \\
\boldsymbol{i}_{\mathrm{T}, \text { unobs }}
\end{array}\right], \quad \boldsymbol{y}_{\varphi}=\boldsymbol{\varphi}_{\mathrm{T}, \text { unobs }}
$$

Thus, complex-valued state variables are obtained by

$$
\underline{\boldsymbol{u}}_{\mathrm{K}, \mathrm{x}}=\boldsymbol{X}_{\mathrm{u}} \mathrm{e}^{\mathrm{j} x_{\delta}}
$$

and

$$
\underline{\boldsymbol{i}}_{\mathrm{T}, \mathrm{y}}=\boldsymbol{Y}_{\mathrm{i}} \mathrm{e}^{\mathrm{j} \boldsymbol{y}_{\varphi}}
$$

respectively. According to Kirchhoff's first law, the two constraints

$$
\underline{\boldsymbol{i}}_{\mathrm{K}, \mathrm{obs}}+\boldsymbol{K}_{\mathrm{KT}, \mathrm{obs}} \underline{\boldsymbol{i}}_{\mathrm{T}, \mathrm{y}}=\mathbf{0}
$$

and

$$
\underline{\boldsymbol{i}}_{\mathrm{K}, \mathrm{obs}}+\boldsymbol{K}_{\mathrm{KT}, \mathrm{obs}} \underline{\boldsymbol{Y}}_{\mathrm{T}, \text { red }} \boldsymbol{K}_{\mathrm{KT}, \text { red }}^{\mathrm{T}} \underline{\boldsymbol{u}}_{\mathrm{K}, \mathrm{x}}=\mathbf{0}
$$

have to be fulfilled. Unfortunately the equation systems of both groups are still underdetermined. But as both groups should describe the same grid state, it is mandatory to match both states, resulting in two additional equations

$$
\underline{\boldsymbol{i}}_{\mathrm{T}, \mathrm{y}}-\underline{\boldsymbol{Y}}_{\mathrm{T}} \boldsymbol{K}_{\mathrm{KT}, \mathrm{Ted}}^{\mathrm{T}} \underline{\boldsymbol{u}}_{\mathrm{K}, \mathrm{x}}=0
$$

and

$$
\underline{\boldsymbol{u}}_{\mathrm{K}, \mathrm{x}}-\underline{\boldsymbol{u}}_{\mathrm{K}}\left(\underline{\boldsymbol{i}}_{\mathrm{T}, \mathrm{y}}\right)=\mathbf{0}
$$

with

$$
\underline{\boldsymbol{u}}_{\mathrm{K}}\left(\underline{\boldsymbol{i}}_{\mathrm{T}, \mathrm{y}}\right)=\left[\begin{array}{c}
\underline{U}_{\text {slack }} \\
\underline{\boldsymbol{Y}}_{\mathrm{KK}, \mathrm{red}, \mathrm{red}}\left(\boldsymbol{K}_{\mathrm{KT}, \text { red }} \underline{\boldsymbol{i}}_{\mathrm{T}, \mathrm{y}}-\underline{\boldsymbol{y}}_{\mathrm{KK}, \text { red,slack }} \underline{U}_{\text {slack }}\right)
\end{array}\right]
$$

$\underline{\boldsymbol{Y}}_{\mathrm{KK}, \mathrm{red}, \mathrm{red}}^{-1}$ is the slack reduced inverse and $\underline{\boldsymbol{y}}_{\mathrm{KK}, \text { red,slack }}$ the slack column of $\underline{\boldsymbol{Y}}_{\mathrm{KK}, \text { red }}$. 
Eq. (83) to Eq. (87) now formulate a nonlinear, overdetermined equation system which can be solved with any least-squares method adequate to conventional power system state estimation.

The result of the reduced grid state calculation is a consistent, complex-valued state vector with all inferior values. For the subsequent steps the following information is needed anyway:

- nodal voltages at central busbars,

- currents of terminals connected to central busbas,

- $\quad$ currents of auxiliary nodes.

Additionally, in some cases voltages of auxiliary nodes might be used.

\subsection{Recursive inference on the original grid state}

In this step the complex-valued grid state of the previous grid without unobserved branches is calculated. Again, the resulting equation system is underdetermined and thus backreferencing is done recursively. The topology and results obtained in chapter 6.3 are referred to as $0^{\text {th }}$ recursion step.

Based on the grid of the previous recursion step, additional nodes are added and a new grid state is calculated until all nodes of all tie-lines are added. Due to nodal voltages at central busbars are already known, tie-lines are again decoupled and can be processed separately. In the following, a recursion step is described.

Adding a node inherits adding at least one line. This line is situated between a previously added node and the new one. Due to the original grid is reconstructed, the node position and line parameters can be obtained directly from $\underline{\boldsymbol{Y}}_{\mathrm{T} \text {,simp }}$.

Depending on the number of nodes still not added to the tie-line, one of the following algorithms is applied.

If only one node has to be added, the last two original line segments defining the position of the node have to be added. The tie-line is now completely reconstructed. The voltage of the new node is the voltage of the auxiliary node of the previous recursion step.

If two nodes have to be added, the last three original line segments have to be added. Thereby, the positions of the new nodes are defined. Due to the fact that voltages and currents of the terminals connected to the outer nodes are entirely known, the transfer functions $\underline{\boldsymbol{H}}$ of the outer lines are used to calculate the voltages of the new nodes.

$$
\left[\begin{array}{l}
\underline{U}_{\mathrm{T} 2} \\
\underline{I}_{\mathrm{T} 2}
\end{array}\right]=\underline{\boldsymbol{H}}_{2,1}\left[\begin{array}{l}
\underline{U}_{\mathrm{T} 1} \\
\underline{I}_{\mathrm{T} 1}
\end{array}\right]
$$

and

$$
\left[\begin{array}{l}
\underline{U}_{\mathrm{T} 5} \\
\underline{I}_{\mathrm{T} 5}
\end{array}\right]=\underline{\boldsymbol{H}}_{5,6}\left[\begin{array}{l}
\underline{U}_{\mathrm{T} 6} \\
\underline{I}_{\mathrm{T} 6}
\end{array}\right]
$$

respectively. The tie-line is now completely reconstructed.

In all other cases to keep the equation system determinable, a maximum amount of three nodes can be processed. So, if there are exactly three nodes to add, they and the respective lines are taken from the original grid. Otherwise, only the two outer lines are taken and all 
other nodes and lines are reduced to two auxiliary lines and one auxiliary node. Considering the outer nodes, which have been determined in the previous recursion step, as nodes 1 and 5 and the new nodes as 2 to 4 , respectively, this results in the following equation system

$$
\left[\begin{array}{ccccc}
\underline{y}_{\mathrm{a}} & \underline{y}_{\mathrm{b}} & & & \\
\underline{y}_{\mathrm{b}} & \underline{y}_{\mathrm{c}} & \underline{y}_{\mathrm{d}} & & \\
& \underline{y}_{\mathrm{d}} & \underline{y}_{\mathrm{e}} & \underline{y}_{\mathrm{f}} & \\
& & \underline{y}_{\mathrm{f}} & \underline{y}_{\mathrm{g}} & \underline{y}_{\mathrm{h}} \\
& & & \underline{y}_{\mathrm{h}} & \underline{y}_{\mathrm{i}}
\end{array}\right]\left[\begin{array}{l}
\underline{U}_{1} \\
\underline{U}_{2} \\
\underline{U}_{3} \\
\underline{U}_{4} \\
\underline{U}_{5}
\end{array}\right]=\left[\begin{array}{c}
\underline{I}_{1} \\
\underline{I}_{2} \\
\underline{I}_{3} \\
\underline{I}_{4} \\
\underline{I}_{5}
\end{array}\right]
$$

$\underline{U}_{1}, \underline{U}_{5}, \underline{I}_{1}$ and $\underline{I}_{5}$ are already known. $\underline{U}_{2}$ and $\underline{U}_{4}$ can be obtained either from the first and last line of Eq. (90) or as well from Eq. (88) and Eq. (89). Thus, Eq. (90) is reformulated

$$
\left[\begin{array}{cccc}
\underline{y}_{\mathrm{d}} & -1 & 0 & 0 \\
\underline{y}_{\mathrm{e}} & 0 & -1 & 0 \\
\underline{y}_{\mathrm{f}} & 0 & 0 & -1
\end{array}\right]\left[\begin{array}{l}
\underline{U}_{3} \\
\underline{I}_{2} \\
\underline{I}_{3} \\
\underline{I}_{4}
\end{array}\right]=-\left[\begin{array}{l}
\underline{y}_{\mathrm{b}} \underline{U}_{1}+\underline{y}_{\mathrm{c}} \underline{U}_{2} \\
\underline{y}_{\mathrm{d}} \underline{U}_{2}+\underline{y}_{\mathrm{f}} \underline{U}_{4} \\
\underline{y}_{\mathrm{g}} \underline{U}_{4}+\underline{y}_{\mathrm{h}} \underline{U}_{5}
\end{array}\right]
$$

Eq. (91) still is underdetermined. But in the previous recursion step, nodes 2 to 4 were summarized as auxiliary node carrying a current $\underline{I}_{\text {aux }}$. Thus, as a constraint

$$
\sum_{\tau=2}^{4} \underline{I}_{\tau}=\underline{I}_{\text {aux }}
$$

This results in a square equation system which is determinable if capacitive shunts are not ignored.

$$
\left[\begin{array}{cccc}
\underline{y}_{\mathrm{d}} & -1 & 0 & 0 \\
\underline{y}_{\mathrm{e}} & 0 & -1 & 0 \\
\underline{y}_{\mathrm{f}} & 0 & 0 & -1 \\
0 & 1 & 1 & 1
\end{array}\right]\left[\begin{array}{l}
\underline{U}_{3} \\
\underline{I}_{2} \\
\underline{I}_{3} \\
\underline{I}_{4}
\end{array}\right]=-\left[\begin{array}{c}
\underline{y}_{\mathrm{b}} \underline{U}_{1}+\underline{y}_{\mathrm{c}} \underline{U}_{2} \\
\underline{y}_{\mathrm{d}} \underline{U}_{2}+\underline{y}_{\mathrm{f}} \underline{U}_{4} \\
\underline{y}_{\mathrm{g}} \underline{U}_{4}+\underline{y}_{\mathrm{h}} \underline{U}_{5} \\
-\underline{I}_{\mathrm{aux}}
\end{array}\right]
$$

If there are more than three nodes to be added, $\underline{I}_{3}$ will be $\underline{I}_{\text {aux }}$ in the next recursion step, otherwise the reconstruction of the tie-line is complete.

\subsection{Recovery of unobserved branch lines}

Due to missing information, the reconstruction of unobserved branch lines can not be done without assumptions. A reasonable way to allocate the load of the remaining node, obtained during recursive back-referencing, to all nodes of the branch is to distribute it proportional to the estimated nodal power which was used to define positions of auxiliary nodes. If this information is not available, other assumptions can be applied i.e. uniformly distributed nodal powers.

Assumptions are solely used for recovery of unobserved branch lines and the determination of auxiliary node positions. Besides the grid reduction approach entirely gets by without any assumed information.

It is obvious, that at unobserved branches the estimation error strongly depends on assumption accuracy. Due to the actual branch state is virtually never correctly assumed, in 
normal case this faulty distribution pattern results in higher errors compared to the state identification process at tie-lines.

\section{Summary and Conclusion}

The behavior and utilization of the electric power system is strongly influenced by the divergent interests of different market participants, particularly in the distribution level. To find a convenient grid operation strategy, decentralized energy management systems are developed.

Knowledge of the grid state is essential for these management systems to work. For historical reasons measurement facilities are sparsely spread in distribution grids. Thus, grid states are not uniquely determinable solely by measured values and have to be estimated using on the one hand supplementary predicted values and on the other hand intelligent methods to infer on probable grid states.

Methods of grid state identification are mathematical algorithms to obtain information on state variables of electric power systems grids. These procedures are based fundamentally on the acquisition of measured, forecasted or estimated values influencing the grid state.

In underdetermined grids the system state is not entirely observed. Therefore, in combination with knowledge of grid topology, equipment parameters and technical constraints, state identification algorithms offer methods to best possibly infer on the actual grid state. They result in a deterministic and consistent state vector.

In this work four different methods of state identification of underdetermined distribution grids are developed and demonstrated using various examples. They vary in the use of available information, the objective and the way, the grid state is determined.

- The boundary load flow algorithm is applied to calculate limits of equipment utilization. Contrarily to the other developed procedures, this method does not return a determined grid state but a set of maximum and minimum terminal current bounds indicating whether equipment is always operated safely or is potentially overstressed. In the latter case grid operation might carry risk and thus, further determining state identification algorithms and an energy management strategy should be applied. So, the boundary load flow may be used as preprocessing method. Furthermore, this algorithm is applicable for power flow decomposition problems and offers information on optimal grid expansion or retreat strategies.

- $\quad$ The second type of grid state identification uses sensitivity analysis to estimate nodal voltages. This method is based on a linearized system model to calculate the influence of all measured values on state variables. Therefore, depending on the coordinate system, different sensitivity matrices are developed determining the linear dependency of nodal voltages on currents and powers. In addition these matrices are used to calculate sensitivity indexes, a valuation of the usefulness of each nodal measurand. Thus, if grid operators aim at improving approximation of grid state, which is only practicable by installing new measuring devices, sensitivity indexes offer information on optimal measurement expansion strategies under technical and economical constraints much faster than conventional optimization problem solvers. Due to the fact that the grid reduction approach offers more accurate state approximations and is not based on a linearized system model, the main field of application of sensitivity analysis is the search for optimal measurands. 
- $\quad$ The availability of measured values is mostly insufficient to uniquely calculate a grid state. Thus, measurands have to be supplemented by a prediction of nodal values. Therefore, active nodal power can be easily forecasted using existing analytic load profiles. Due to reactive power profiles have not been available so far, they are developed in this work based on measured time-series of hand-over points to superior grids within several years. Measured nodal powers and power estimations at each unobserved node are merged to a complete and complex-valued nodal power vector which can be used to obtain the grid state by power flow calculation. This method should be applied in case of utilization of the grid reduction approach is not possible due requirements are not fulfilled, only a few measurands are missing in the nodal power vector and the time-dependent behavior of loads is roughly predictable.

- $\quad$ The final grid state identification method which is called grid reduction approach uses both measured nodal and terminal values as input variables. Contrarily to the method based on sensitivity analysis this approach extensively exploits typical properties of distribution grid topology and thus is able to more accurately estimate the grid state even without installation of new measurement facilities which is a further advantage. To estimate nodal voltages, the grid is reduced to the observed central busbars and a minimum of auxiliary nodes on remaining tie-lines. Thereby the equation system size is reduced until the system is solvable. After the reduced grid state is calculated a recursive algorithm is applied to back-reference to the original grid by re-adding a small number of nodes in each recursion step. The obtained results are more accurate than the ones obtained by sensitivity analysis. Furthermore, the grid reduction approach does not need a linearized model and thus approximation error is independent of the deviation from an assumed operation point.

It is clearly demonstrated that state identification methods are of practical importance for grid operators but as well for grid planners. In the latter case especially the boundary load flow method is valuable to indicate necessary grid modifications and to find cost-effective strategies of expansion, maintenance and even retreat. Additionally, this method helps making decisions where to connect new loads or DG sources. Furthermore, grid planners may apply sensitivity analysis to identify significant influencing variables and to expand the measurement topology accordingly.

On the other side, during operating conditions knowledge of the grid state is essential for grid control systems. On the one hand, the generated reactive power profiles offer the ability of entire long-term forecasting of load situations to local utility operators and on the other hand particularly the grid reduction approach returns close approximations of the current actual system state which is used for resource scheduling and allocation.

Thus, it is obvious that state identification of distribution grids offers an important contribution to face the challenges of future distributed energy supply and establish a sound basis for highly automated decentralized energy management systems. 


\section{References}

Abur, A.; Expósito, A. G.: Power system state estimation: theory and implementation, Dekker, New York, 2004.

Albert, A.: Regression and the Moore-Penrose Pseudoinverse, Academic Press Inc., New York, London, 1972.

Dimitrovski, A.; Tomsovic, K.: Boundary Load Flow Solutions, IEEE Transactions of Power Systems, Vol. 19, No. 1, February 2004.

EWE AG (Hrsg.): 10 Bullensee-Thesen und abgeleitete Handlungsempfehlungen zur zukünftigen Energieversorgung (3. Auflage), Oldenburg, 2007.

Meier, H.; Fünfgeld, C.; Adam, C.; Schieferdecker, B.: Repräsentative VDEW-Lastprofile, VDEW, Frankfurt (Main), 1999.

Merkt, B.: Beitrag zur Zustandsidentifikation von elektrischen Energieversorgungsnetzen, Dissertation, Leibniz Universität Hannover - Institut für Energieversorgung und Hochspannungstechnik - Fachgebiet Elektrische Energieversorgung, Hannover, August 2007.

Oeding, D.; Oswald, B. R.: Elektrische Kraftwerke und Netze (6. Auflage), Springer, Berlin, 2004

Reese, C.: Beitrag zur Entwicklung eines dezentralen Energiemanagementsystems, Diplomarbeit DA-473, Leibniz Universität Hannover - Institut für Energieversorgung und Hochspannungstechnik - Fachgebiet Elektrische Energieversorgung, Hannover, October 2007.

VDEW (Hrsg.): Repräsentative VDEW-Lastprofile, VDEW-Verlag, Frankfurt (Main), 1999.

Walters, H.; Parker, L. R.; Morgan, S. L.; Deming, S. N.: Sequential Simplex Optimization, CRC Press, Boca Raton, 2000.

Wang, Z; Alvarado, F. L.: Interval Arithmetic in Power Flow Analysis, Proceedings of the IEEE International Conference on Power Industry Computer Application, May 1991.

Wolter, M.: A new approach on state identification of underdetermined MV grids based on grid reduction, Proceedings of the 4th IASTED Asian Conference on Power \& Energy Systems (AsiaPES2008), Langkawi, Malaysia, April 2008.

Wolter, M.: Cost-effective grid state identification of mean voltage grids by using Sensitivity Analysis, Proceedings of the 7th IASTED International Conference on Power and Energy Systems (EuroPES 2007), Spain, August 2007.

Wolter, M.: Entwicklung eines MicroGrid Simulator, Diplomarbeit DA-469, Leibniz Universität Hannover - Institut für Energieversorgung und Hochspannungstechnik - Fachgebiet Elektrische Energieversorgung, Hannover, August 2006.

Wolter, M.: Load type mixture extraction from measured time series of hand-over point active power, Proceedings of the 7th IASTED International Conference on Power and Energy Systems (EuroPES 2007), Spain, August 2007.

Wolter, M.: Grid State identification of distribution grids, Shaker Verlag, Aachen, 2008.

Wolter, M.: Grid reduction approach for state identification of distribution grids, Proceedings of the IEEE PES Annual General Meeting 2009, Calgary, Canada, September 2009. 


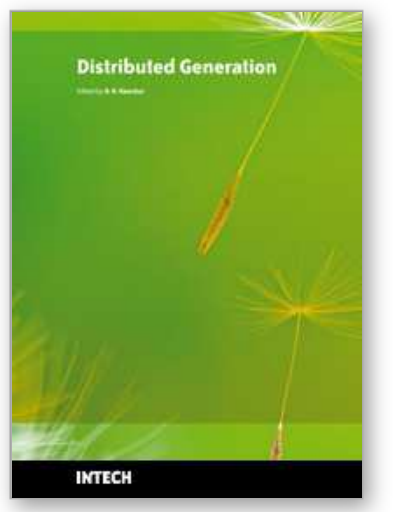

\author{
Distributed Generation \\ Edited by D N Gaonkar
}

ISBN 978-953-307-046-9

Hard cover, 406 pages

Publisher InTech

Published online 01, February, 2010

Published in print edition February, 2010

In the recent years the electrical power utilities have undergone rapid restructuring process worldwide. Indeed, with deregulation, advancement in technologies and concern about the environmental impacts, competition is particularly fostered in the generation side, thus allowing increased interconnection of generating units to the utility networks. These generating sources are called distributed generators (DG) and defined as the plant which is directly connected to distribution network and is not centrally planned and dispatched. These are also called embedded or dispersed generation units. The rating of the DG systems can vary between few $\mathrm{kW}$ to as high as $100 \mathrm{MW}$. Various new types of distributed generator systems, such as microturbines and fuel cells in addition to the more traditional solar and wind power are creating significant new opportunities for the integration of diverse DG systems to the utility. Interconnection of these generators will offer a number of benefits such as improved reliability, power quality, efficiency, alleviation of system constraints along with the environmental benefits. Unlike centralized power plants, the DG units are directly connected to the distribution system; most often at the customer end. The existing distribution networks are designed and operated in radial configuration with unidirectional power flow from centralized generating station to customers. The increase in interconnection of $D G$ to utility networks can lead to reverse power flow violating fundamental assumption in their design. This creates complexity in operation and control of existing distribution networks and offers many technical challenges for successful introduction of DG systems. Some of the technical issues are islanding of DG, voltage regulation, protection and stability of the network. Some of the solutions to these problems include designing standard interface control for individual DG systems by taking care of their diverse characteristics, finding new ways to/or install and control these DG systems and finding new design for distribution system. DG has much potential to improve distribution system performance. The use of DG strongly contributes to a clean, reliable and cost effective energy for future. This book deals with several aspects of the DG systems such as benefits, issues, technology interconnected operation, performance studies, planning and design. Several authors have contributed to this book aiming to benefit students, researchers, academics, policy makers and professionals. We are indebted to all the people who either directly or indirectly contributed towards the publication of this book.

\title{
How to reference
}

In order to correctly reference this scholarly work, feel free to copy and paste the following:

Martin Wolter (2010). State Identification of Underdetermined Grids, Distributed Generation, D N Gaonkar (Ed.), ISBN: 978-953-307-046-9, InTech, Available from: http://www.intechopen.com/books/distributedgeneration/state-identification-of-underdetermined-grids 
open science | open minds

InTech Europe

University Campus STeP Ri

Slavka Krautzeka 83/A

51000 Rijeka, Croatia

Phone: +385 (51) 770447

Fax: +385 (51) 686166

www.intechopen.com
InTech China

Unit 405, Office Block, Hotel Equatorial Shanghai

No.65, Yan An Road (West), Shanghai, 200040, China

中国上海市延安西路65号上海国际贵都大饭店办公楼 405 单元

Phone: +86-21-62489820

Fax: +86-21-62489821 
(C) 2010 The Author(s). Licensee IntechOpen. This chapter is distributed under the terms of the Creative Commons Attribution-NonCommercialShareAlike-3.0 License, which permits use, distribution and reproduction for non-commercial purposes, provided the original is properly cited and derivative works building on this content are distributed under the same license. 